\author{
Xiaofei Liu, PhD Candidate \\ School of Economics and Management \\ Beijing Jiaotong University, China \\ E-mail: 18611989172@163.com \\ Lecturer Wenrui Li (Corresponding author) \\ School of Economics and Management \\ Beijing Jiaotong University, China \\ E-mail: 15501088811@163.com
}

\title{
EVALUATING THE DEVELOPMENT EFFICIENCY OF CULTURAL INDUSTRY BY A BILATERAL SFA MODEL
}

\begin{abstract}
In this paper, the Stochastic Frontier Analysis model is used to measure the technical efficiency and its influencing factors of cultural industry in the Central China. The results show that the technical efficiency of the cultural industry in Central China has been at a low level, and the most areas have maintained a good growth trend. The technical efficiency level of the interregional cultural industry is significantly different.

Keywords: Central China; cultural industry; technical efficiency; stochastic frontier analysis.
\end{abstract}

\section{JEL Classification: Z1, C7}

\section{Introduction}

A major strategic goal of deepening cultural system reform and building a strong socialist cultural power was put forward by the 18th National Congress of the Communist Party of China. In recent years, the cultural industries have developed rapidly in the Central China, but the current development level of the cultural industries is not high, and there is a significant difference in structural imbalance of different cultural industries. Moreover, the development of the cultural industries in Central China showed a downward trend for the first time. Therefore, accurate analysis of the current situation of the cultural industry development efficiency in Central China, measurement of relevant factors' impact on the development of the cultural industries and exploration of an effective path for improving the development efficiency of the cultural industries are of great practical significance for accelerating regional cultural industry development to promote economic growth.

The Stochastic Frontier Analysis model is widely used for efficiency analysis 
in different industries. Evgallıoglu et al. used the data from 55 natural gas distribution companies in Turkey during 2013 and 2015 to examine technical efficiencies[1].Ahmad disaggregated and explored the components of efficiency that contribute to the change in output, scale of production and technical efficiency of sugar mills in Uttar Pradesh and data of 115 sugar mills for the year 2011-2012 was collected[2]. Prosset al. used a Bayesian Stochastic Frontier Analysis (SFA) model to assess hospital performance along the dimensions of resources and quality of stroke care in German hospitals[3].A few researchers focus on culturally relevant areas, for examples:Herrero-Prietoet al. evaluated the technical efficiency of regions in attracting greater flows of cultural tourism considering their own cultural resources available in the medium term[4].Figueroa et al. used a two stage model, estimating regional performance using data envelopment analysis and analyzing the impact of analyzing the impact of external variables on efficiency applying bootstrap techniques and truncated regression models[5].

As a high-efficiency, pollution-free sunrise industry, the cultural industry has received a lot of attention and has been vigorously developed. There are many researches on the development efficiency of the cultural industries in China, but only a few are about that of the Central China . At present, the research objects can be mainly divided into two categories. The first category includes listed companies in the cultural industries, which are used as the samples for studying the development efficiency of the cultural industries. For example, in 2013, Le et al. used the stochastic frontier analysis KSS model to measure and analyze the development efficiency of the cultural enterprises in China. The research found that the development efficiency of the cultural industries in China was still at a low level and capital inputting played a leading role in the development of the cultural industries in China[6]. In 2015, Zhao et al. measured and analyzed the development efficiency and influencing factors of the cultural and creative industries in China. The research found that the development efficiency value of cultural and creative enterprises was generally low and financing cost was the most important factor affecting the development efficiency of enterprises[7]. The other category refers to the research on regional cultural industries. For example, in 2012, Dong et al. used the SFA model to empirically analyze the data of 31 provinces from 2004 to 2009 and found that the market culture demand played a significant role in promoting the improvement of the cultural output efficiency[8]; in 2012, Ma et al. used the SFA model to analyze the efficiency and influencing factors of the cultural industries in China and found that the improvement of residents' living standard had a significant effect on the efficiency of the cultural industries in China[9]; in 2015, Zhao et al. used the SFA model to regionally divide the panel data of 30 provinces and cities in China and investigated the degree of various factors' influence on the development efficiency of regional

Evaluating the Development Efficiency of Cultural Industry by a Bilateral SFA Model cultural industries in China, and found that the level of average development efficiency of the cultural industries in China was low with a significant difference 
between regions and the regional relative cultural market demand had a significant impact on the development efficiency of the cultural industries in China[10].

The above literature provides a solid theoretical and empirical basis for further studying the development efficiency of the cultural industries, but there is still room for further improvement. From the perspective of the research subject, most of the literature mainly analyzes the development efficiency of the cultural industries at the level of listed companies and it is lack of analysis at the overall macro level of a region, which is not conducive to our understanding of the overall development of inter-provincial cultural industries. As a result, it leads to poor pertinence and policy guidance of the preliminary research. This paper focuses on the inter-provincial regions to analyze the development efficiency and regional differences of the regional cultural industries, and adopts the stochastic frontier analysis model to evaluate and analyze the development efficiency of the regional cultural industries in the Central China based on the panel data of prefecture-level cities from 2003 to 2016, aiming to evaluate the development efficiency of the cultural industries of various prefecture-level cities in the Central China and provide policy guidance for further improving the development efficiency of the cultural industries in the Central China and promoting the sustainable and healthy development of the cultural industries.

\section{Basic SFA model}

Stochastic Frontier Analysis (SFA) is widely used to measure the economic development efficiency of regions or enterprises. The model is determined by the function of $\ln Y_{i}=x_{i} \beta+V_{i}-U_{i}$ and the upper bound of its output value is a random variable of $\exp \left(x_{i} \beta+V_{i}\right)$. The model as follows[11-12]:

$y_{i t}=f\left(x_{i t}, \beta\right) \exp \left(V_{i t}-U_{i t}\right), i=1, \ldots, N, t=1, \ldots, T$

In the above formula, $y_{i t}$ indicates the output of no. $i$ decision making unit (DMU) in the no. ${ }^{t}$ period. ${ }^{x_{i t}}$ indicates the input of various factors of no. ${ }^{i} \mathrm{DMU}$ in the no. ${ }^{t}$ period. ${ }^{\beta}$ indicates the parameters to be estimated. $V_{i t}$ refers to the stochastic error term, indicating the influence of stochastic disturbance and obeying the normal distribution $N\left(0, \sigma_{v}^{2}\right) . U_{i t}$ indicates the non-negative random variable of the inefficient part, obeying the normal distribution $N^{+}\left(u, \sigma_{i t}^{2}\right)$.

$$
T E_{i t}=\exp \left(-U_{i t}\right)=\frac{E\left[f\left(x_{i t}, \beta\right) \exp \left(V_{i t}-U_{i t}\right)\right]}{E\left[f\left(x_{i t}, \beta\right) \exp \left(V_{i t}\right)\right]}
$$

In formula (2), $T E$ indicates the development efficiency and it is the ratio of actual output expectation to production frontier expectation. When $U_{i t}=0, T E=1$ and DMU is above the production frontier, it shows that technology is completely effective; when $U_{i t}>0$, DMU is below the production frontier, it shows that technology is ineffective. In order to further study the difference in development 


\section{Xiaofei Liu, Wenrui Li}

efficiency, Battese et al. added a non-efficiency function in the extended model and used the maximum likelihood estimation in 1995, as shown in formula (3):

$$
U_{i t}=z_{i t} \delta+w_{i t}
$$

In formula (3), $U_{i t}$ indicates inefficiency (efficiency loss); $z_{i t}$ indicates the factor that causes inefficiency; $\delta$ indicates the parameter to be estimated, which indicates that it has a positive effect on efficiency when $\delta<0$ and that it has a reverse effect on efficiency when $\delta>0$.

According to the above assumption, the following distribution density function can

be obtained:

$$
f\left(u_{t}\right)=\left(\frac{2}{\sqrt{2 \pi \sigma_{u}^{2}}}\right)^{N} \exp \left(-\frac{u_{t} u_{t}}{2 \sigma_{u}^{2}}\right)
$$

$f\left(u_{t}, v_{t}\right)=\left(\frac{1}{\pi \sigma_{u} \sigma_{v}}\right)^{N}|\Sigma|^{-\frac{1}{2}} \exp \left(-\frac{u_{t}^{\prime} u_{t}}{2 \sigma_{u}^{2}}-\frac{v_{t}^{\prime} \Sigma^{-1} v_{t}}{2 \sigma_{v}^{2}}\right)$

Suppose $\varepsilon_{t}=v_{t}-u_{t}$, the joint distribution density function of $\left(u_{t}, \varepsilon_{t}\right)$ is:

$$
\begin{aligned}
& f\left(u_{t}, \varepsilon_{t}\right)=\left(\frac{1}{\pi \sigma_{u} \sigma_{v}}\right)^{N}|\Sigma|^{-\frac{1}{2}} \exp \left(-\frac{u_{t}^{\prime} u_{t}}{2 \sigma_{u}^{2}}-\frac{\left(\varepsilon_{t}+u_{t}\right) \Sigma^{-1}\left(\varepsilon_{t}+u_{t}\right)}{2 \sigma_{v}^{2}}\right) \\
& =\left(\frac{1}{\pi \sigma_{u} \sigma_{v}}\right)^{N}|\Sigma|^{-\frac{1}{2}} \exp \left(-\frac{1}{2}\left(u_{t}-\mu_{t}\right)^{\prime} \Omega^{-1}\left(u_{t}-\mu_{t}\right)\right) \exp \left(-\frac{1}{2} \varepsilon\left(\sigma_{u}^{2} I+\sigma_{v}^{2} \Sigma\right)^{-1} \varepsilon_{t}\right)
\end{aligned}
$$

where

$$
\begin{aligned}
& \mu_{t}=-\sigma_{u}^{2}\left[\sigma_{u}^{2} I+\sigma_{v}^{2} \Sigma\right]^{-1} \varepsilon_{t} \\
& \Omega=\sigma_{t}^{2}\left[I-\sigma_{u}^{2}\left(\sigma_{u}^{2} I+\sigma_{v}^{2} \Sigma\right)^{-1}\right]
\end{aligned}
$$

The distribution density function is obtained: 
$f\left(\varepsilon_{t}\right)=\int_{0}^{\infty} f\left(u_{t}, \varepsilon_{t}\right) d u_{t}$

$=\left(\frac{1}{\pi \sigma_{u} \sigma_{v}}\right)^{N}|\Sigma|^{-\frac{1}{2}} \exp \left(-\frac{1}{2} \varepsilon_{t}\left(\sigma_{u}^{2} I+\sigma_{v}^{2} \Sigma\right)^{-1} \varepsilon_{t}\right) \int_{0}^{\infty} \exp \left(-\frac{1}{2}\left(u_{t}-\mu_{t}\right)^{\prime} \Omega^{-1}\left(u_{t}-\mu_{t}\right)\right) d u_{t}$

$=\left(\frac{2}{\sqrt{2 \pi}}\right)^{N}\left|\sigma_{u}^{2} I+\sigma_{v}^{2} \Sigma\right|^{-\frac{1}{2}} \exp \left(-\frac{1}{2} \varepsilon_{t}\left(\sigma_{u}^{2} I+\sigma_{v}^{2} \Sigma\right)^{-1} \varepsilon_{t}\right) \Phi\left(\Omega^{-\frac{1}{2}} \mu\right)$

where $\Phi(\circ)$ is the standard normal distribution function of multiple variables.

$\Omega^{-\frac{1}{2}} \mu_{t}=-\frac{\sigma_{u}}{\sigma_{v}}\left(\sigma_{u}^{2} I+\sigma_{v}^{2} \Sigma\right)^{-\frac{1}{2}} \Sigma^{-\frac{1}{2}} \varepsilon_{t}$

Based on equation (11), the logarithmic likelihood function of the model can be obtained:

$$
\begin{aligned}
& \prod\left(\alpha \cdot \beta, \sigma_{u}, \sigma_{v}, \lambda, \rho\right)=N T \ln (2)-\frac{N T}{2} \ln (2 \pi)-\frac{T}{2} \ln \left(\left|\sigma_{u}^{2} I+\sigma_{v}^{2} \Sigma\right|\right) \\
& -\frac{1}{2} \sum_{t=1}^{T} \varepsilon_{t}\left[\sigma_{u}^{2} I+\sigma_{v}^{2} \Sigma\right]^{-1} \varepsilon_{t}+\sum_{t=1}^{T} \ln \left[\Phi\left(\Omega^{-\frac{1}{2}} \mu_{t}\right)\right]
\end{aligned}
$$

By maximizing the logarithmic likelihood function equation (12), the parameter estimation of the above model can be obtained.

\section{The improved model}

The traditional efficiency analysis model can only calculate the efficiency value based on input and output, but does not consider other factors affecting efficiency. This paper further introduces the bilateral stochastic frontier analysis model and adds some factors that affect the development efficiency of the cultural industries on the basis of input and output, so that the development efficiency of the cultural industries can be more comprehensively calculated. Moreover, the paper also fully considers the impact of some unobservable factors on the development efficiency of the cultural industries. The model used in the paper is set as follows.

$$
\begin{aligned}
\text { culture }_{i t} & =i\left(x_{i t}\right)+\omega_{i t}-u_{i t}+\varepsilon_{i t} \\
& =i\left(x_{i t}\right)+\xi_{i t} \\
& =x_{i t} \delta+\xi_{i t}
\end{aligned}
$$

Where, xit indicates a series of variables affecting the development efficiency of the cultural industries, including economic development level, industrial structure, human capital level, urbanization, innovation level, degree of openness, financial expenditure level, infrastructure, investment rate, etc. $\delta$ indicates the parameter 


\section{Xiaofei Liu, Wenrui Li}

estimation vector, and $i(x i t)$ indicates the level of development efficiency of the frontier cultural industries, i.e. the level of development efficiency of the cultural industries under the established influence. In the formula of $i(x i t)=\lambda x_{i t}, \lambda$ indicates the characteristic variable estimation parameter. In the compound residual term of $\xi_{i t}=\omega i t-u i t+\varepsilon i t$, Eit indicates the stochastic error term, reflecting the deviation of the development efficiency of the cultural industries from the stochastic efficiency level caused by the unobservable factors. Wit is used to describe the positive effect of unobservable factors on the development efficiency of the cultural industries, and $\omega i t \geq 0$. uit is used to describe the negative effect of unobservable factors on the development efficiency of the cultural industries, and uit $\geq 0$. When $\omega_{i t}=0$, it is only affected by the negative effect of the unobservable factors, and when $u i t=0$, it is only affected by the positive effect of the unobservable factors. When either of the above two cases occurs, it indicates the unilateral stochastic frontier model. When the two are both 0 , it indicates the general OLS model. Because the compound residual term of $\xi_{\text {it }}$ may not be 0 , it will cause the OLS model to generate biased estimate. It can be learned from formula (1) that the ultimate realization of the actual development efficiency of the cultural industries is a result of the bilateral effect of the negative and positive effects of the unobservable factors: the positive effect of the unobservable factors on the efficiency level makes the development efficiency of the cultural industry higher than the frontier efficiency level, while the negative effect caused by the unobservable factors makes the actual development efficiency of the cultural industries lower than the development efficiency of the frontier cultural industries. The deviation degree of the actual development efficiency of the cultural industries is measured by calculating the net effect influenced by the two together. In the case of biased estimate by the OLS model, the maximum likelihood estimation method can be used to obtain an effective estimation result. Therefore, we need to make the following assumptions about the residual distribution: the stochastic error term of Eit obeys normal distribution, i.e. $\operatorname{Eit} \sim \operatorname{iidN}\left(0, \sigma_{\varepsilon}^{2}\right)$; $\omega_{i t}$ and uit both obey exponential distribution, i.e. $\omega_{i t} \sim \operatorname{iidEXP}\left(\sigma_{\omega}, \sigma_{\omega}^{2}\right)$ and $u i t \sim \operatorname{iidEXP}\left(\sigma_{u}, \sigma_{u}^{2}\right)$. Moreover, the error items are independent of each other and not related to regional characteristics of $x_{i t}$. On the basis of the above distribution assumptions, the probability density function of the compound residual term of $\xi_{i t}$ can be further derived:

$f\left(\xi_{i t}\right)=\frac{\exp \left(\alpha_{i t}\right)}{\sigma_{u}+\sigma_{\omega}} \Phi\left(\gamma_{i t}\right)+\frac{\exp \left(\beta_{i t}\right)}{\sigma_{u}+\sigma_{\omega}} \int_{-\eta i t}^{\infty} \varphi(x) d x=\frac{\exp \left(\alpha_{i t}\right)}{\sigma_{u}+\sigma_{\omega}} \Phi\left(\gamma_{i t}\right)+\frac{\exp \left(\beta_{i t}\right)}{\sigma_{u}+\sigma_{\omega}} \varphi\left(\eta_{i t}\right)$

Where, $\Phi(\bullet)$ and $\phi(\bullet)$ are respectively the cumulative distribution function and probability density function of the standard normal distribution. Other parameters 
are set as follows:

$\alpha_{i t}=\frac{\sigma_{v}^{2}}{2 \sigma_{\omega}^{2}}+\frac{\xi_{i}}{\sigma_{\omega}} \beta_{i t}=\frac{\sigma_{v}^{2}}{2 \sigma_{u}^{2}}-\frac{\xi_{i}}{\sigma_{u}} \gamma_{i t}=-\frac{\xi_{i t}}{\sigma_{v}}-\frac{\sigma_{v}}{\sigma_{u}} \eta_{i t}=\frac{\xi_{i t}}{\sigma_{v}}-\frac{\sigma_{v}}{\sigma_{\omega}}$

Based on the above parameter estimations, in the non observed value samples, the estimated maximum likelihood function can be expressed as:

$\ln L(X ; \pi)=-n \ln \left(\sigma_{\omega}+\sigma_{u}\right)+\sum_{i=1}^{n} \ln \left[e^{\alpha_{i t}} \Phi\left(\gamma_{i t}\right)+e^{\beta i t} \Phi\left(\eta_{i t}\right)\right]$

Where, $\pi=\left[\beta, \sigma_{v}, \sigma_{\omega}, \sigma_{u}\right]$. The likelihood function is further maximized (16) to finally obtain all parameter values of the maximum likelihood estimation. In addition, it is necessary to estimate the $\omega_{i t}$ and uit. Therefore, the condition density function of the two is further derived:

$$
\begin{aligned}
& f\left(\omega_{i t} \mid \xi_{i t}\right)=\frac{\left(1 / \sigma_{u}+1 / \sigma_{\omega}\right) \exp \left[-\left(1 / \sigma_{u}+1 / \sigma_{\omega}\right) \omega_{i t}\right] \Phi\left(\omega_{i t} / \sigma_{\nu}+\eta_{i t}\right)}{\exp \left(\beta_{i t}-\alpha_{i t}\right)\left[\Phi\left(\eta_{i t}\right)+\exp \left(\alpha_{i t}-\beta_{i t}\right) \Phi\left(\gamma_{i t}\right)\right]} \\
& f\left(u_{i t} \mid \xi_{i t}\right)=\frac{\left(1 / \sigma_{u}+1 / \sigma_{\omega}\right) \exp \left[-\left(1 / \sigma_{u}+1 / \sigma_{\omega}\right) u_{i t}\right] \Phi\left(u_{i t} / \sigma_{\nu}+\eta_{i t}\right)}{\Phi\left(\eta_{i t}\right)+\exp \left(\alpha_{i t}-\beta_{i t}\right) \Phi\left(\gamma_{i t}\right)}
\end{aligned}
$$

Based on formulas (17) and (18), the conditional expectation of $\omega i t$ and uit can be estimated:

$$
\begin{aligned}
& E\left(\omega_{i t} \mid \xi_{i t}\right)=\frac{1}{\left(1 / \sigma_{u}+1 / \sigma_{\omega}\right)}+\frac{\sigma_{v}\left[\Phi\left(-\eta_{i t}\right)+\eta_{i t} \Phi\left(\eta_{i t}\right)\right]}{\exp \left(\beta_{i t}-\alpha_{i t}\right)\left[\Phi\left(\eta_{i t}\right)+\exp \left(\alpha_{i t}-\beta_{i t}\right) \Phi\left(\gamma_{i t}\right)\right]} \\
& E\left(u_{i t} \mid \xi_{i t}\right)=\frac{1}{\left(1 / \sigma_{u}+1 / \sigma_{\omega}\right)}+\frac{\exp \left(\alpha_{i t}-\beta_{i t}\right) \sigma_{v}\left[\Phi\left(-\gamma_{i t}\right)+\eta_{i t} \Phi\left(\gamma_{i t}\right)\right]}{\left.\Phi\left(\eta_{i t}\right)+\exp \left(\alpha_{i t}-\beta_{i t}\right) \Phi\left(\gamma_{i t}\right)\right]}
\end{aligned}
$$

By using formulas (19) and (20), we can estimate the absolute degree of the efficiency levels of enterprises facing positive and negative effects deviating from the frontier efficiency level. In order to facilitate comparison, it is necessary to further converting the absolute degree into the percentages of the positive and negative effects of unobservable factors being higher or lower than the frontier level. The specific formula is as follows:

$$
\begin{aligned}
& E\left(1-\mathrm{e}^{-\omega_{i t}} \mid \xi_{i t}\right)=1-\frac{\left(1 / \sigma_{u}+1 / \sigma_{\omega}\right)\left[\Phi\left(\gamma_{i t}\right)+\exp \left(\beta_{i t}-\alpha_{i t}\right) \exp \left(\sigma_{v}^{2} / 2-\sigma_{v} \eta_{i t}\right) \Phi\left(\eta_{i t}-\sigma_{v}\right)\right]}{\left[1+\left(1 / \sigma_{u}+1 / \sigma_{\omega}\right)\right] \exp \left(\beta_{i t}-\alpha_{i t}\right)\left[\Phi\left(\eta_{i t}\right)+\exp \left(\alpha_{i t}-\beta_{i t}\right) \Phi\left(\gamma_{i t}\right)\right]} \\
& E\left(1-\mathrm{e}^{-u i t} \mid \xi_{i t}\right)=1-\frac{\left(1 / \sigma_{u}+1 / \sigma_{\omega}\right)\left[\Phi\left(\eta_{i t}\right)+\exp \left(\alpha_{i t}-\beta_{i t}\right) \exp \left(\sigma_{\nu}^{2} / 2-\sigma_{v} \gamma_{i t}\right) \Phi\left(\gamma_{i t}-\sigma_{v}\right)\right]}{\left[1+\left(1 / \sigma_{u}+1 / \sigma_{\omega}\right)\right]\left[\Phi\left(\eta_{i t}\right)+\exp \left(\alpha_{i t}-\beta_{i t}\right) \Phi\left(\gamma_{i t}\right)\right]}
\end{aligned}
$$

We can further use the formulas (21) and (22) to derive the net effect of the 


\section{Xiaofei Liu, Wenrui Li}

positive and negative effects of unobservable factors influencing the development efficiency of the cultural industries:

$$
N E=E\left(1-\mathrm{e}^{-\omega_{i t}} \mid \xi_{i t}\right)-E\left(1-\mathrm{e}^{-u_{i t}} \mid \xi_{i t}\right)=E\left(\mathrm{e}^{-u i t}-\mathrm{e}^{-\omega_{i t}} \mid \xi_{i t}\right)
$$

\section{Case study and discussion}

\subsection{The data}

In view of the availability of data, the output variable mentioned in this paper refers to the added value of the cultural industries, the variable of capital element input refers to the fixed asset investment in the cultural industries, and the labor element variable refers to the number of practitioners in the cultural industries. Other factors affecting the cultural industries include the economic development level expressed by per capita GDP, the industrial structure measured by the ratio of output value of the tertiary industry to output value of the secondary industry, the human capital level measured by the percapita years of being educated, the urbanization measured by the proportion of urban population in total population, the level of innovation measured by the number of patents granted, the degree of openness measured by the proportion of total imports and exports in GDP, the level of fiscal expenditure measured by the proportion of fiscal expenditure in GDP, and infrastructure expressed by highway mileages per 10,000 persons.

\section{2 the results}

This paper estimates the development efficiency of the cultural industries based on the bilateral stochastic frontier analysis model. The model estimation results are shown in Table 1. The first column shows the estimation results in the least square method and the latter six models show the maximum likelihood estimation results to estimate the bilateral stochastic frontier analysis model. Model 0 does not control time effect or region effect; model 1 and model 2 gradually control time effect and region effect; and model 3 and model 5 gradually control the positive and negative effects of the unobservable factors. From the perspective of the maximum likelihood value and the maximum likelihood ratio, the estimation results obtained through model 5 should be selected as the model for estimating the development efficiency of the cultural industries. As shown by the results in table 1, cultural capital and labor have both significantly improved the development efficiency of the cultural industries in all models. At the same time, other influencing factors have all improved the development efficiency of the cultural industries in terms of high significance, indicating that the model has better estimation results.

Table 1. Basic estimation results of the bilateral stochastic frontier analysis model

\begin{tabular}{llllllll}
\hline Period fixed effect & YES & NO & YES & YES & YES & YES & YES \\
\hline Provincial fixed effect & YES & NO & NO & YES & YES & YES & YES \\
\hline Sample size & 168 & 168 & 168 & 168 & 168 & 168 & 168 \\
\hline Logarithmic likelihood & 3978.4 & 3845.2 & 4093.1 & 5798.4 & 6530.5 & 7891.4 & 7993.5 \\
\hline
\end{tabular}


Evaluating the Development Efficiency of Cultural Industry by a Bilateral SFA Model

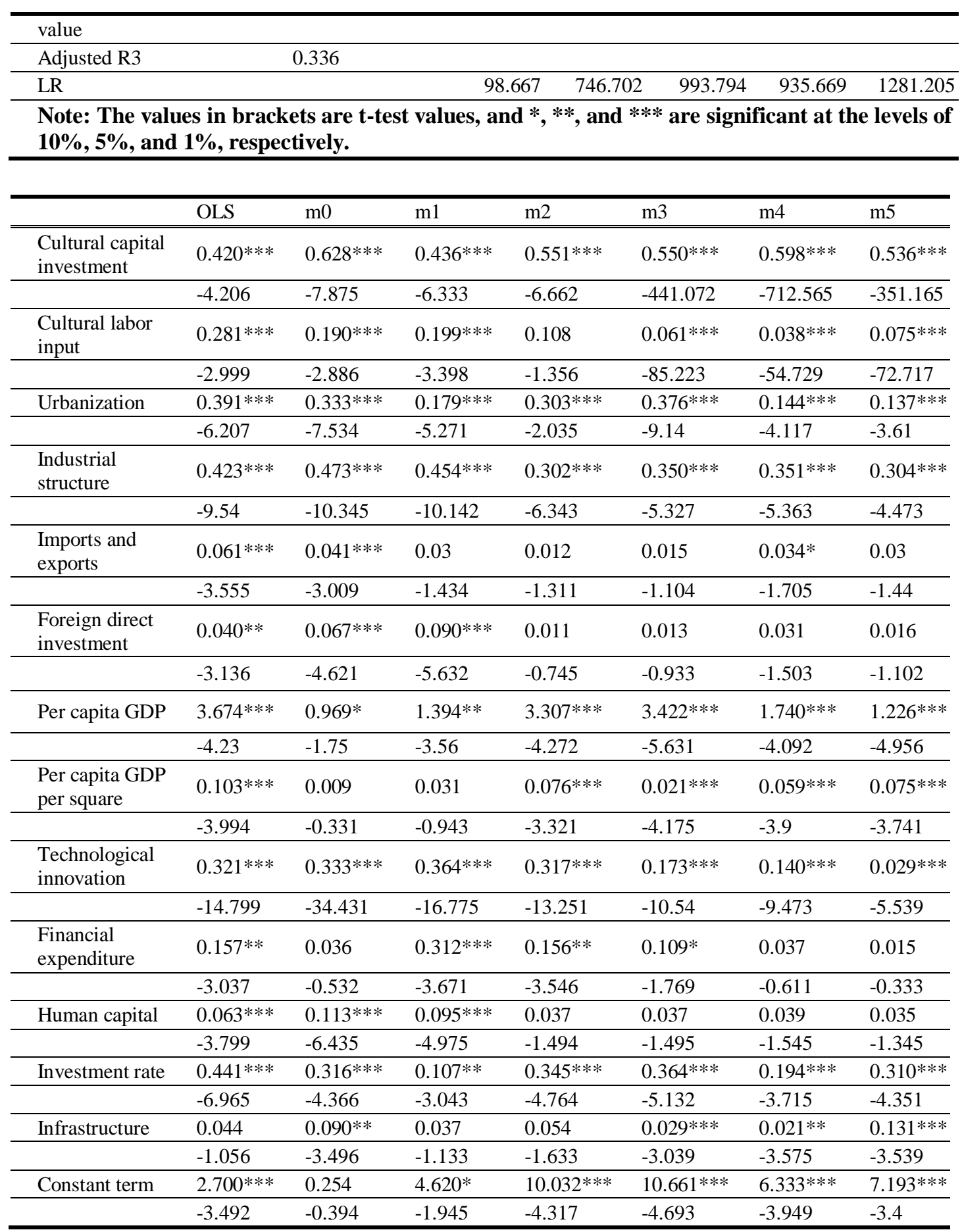




\section{Xiaofei Liu, Wenrui Li}

\subsection{Discussion: A case study of Hubei Province}

Hubei Province is a typical province with the characteristics of the Central China. This paper will develop improved SFA model to measure the technical efficiency and its influencing factors of cultural industry in Hubei Province ,the sampling period of the research of this paper is from 2003 to 2016, covering 12 prefecture-level cities in Hubei Province. Data used in this paper is from official documents such as Hubei Statistical Yearbook from 2004 to 2017 and statistical bulletins of local governments of various prefecture-level cities.

According to statistics, as of the end of 2015, the total output value of the cultural industries in Hubei was RMB 8.654 billion. From the perspective of industrial composition, the added value of the art industry has increased by RMB 81.14 million compared with last year, showing a year-on-year growth of $13.49 \%$; the added value of library industry has increased by RMB 66.62 million compared with last year, showing a year-on-year growth of $24.06 \%$; that of the mass cultural industry has increased by RMB 131 million, showing a year-on-year growth of $40.94 \%$. Compared with last year, the added value of the art education industry has decreased by RMB 0.46 million, showing a year-on-year decline of less than $0.01 \%$; and that of cultural market operators has decreased by RMB 1.342 billion, showing a year-onyear decline of $22.70 \%$.

\begin{tabular}{llllllll}
\hline Period fixed effect & YES & NO & YES & YES & YES & YES & YES \\
\hline Provincial fixed effect & YES & NO & NO & YES & YES & YES & YES \\
\hline Sample size & 168 & 168 & 168 & 168 & 168 & 168 & 168 \\
\hline $\begin{array}{l}\text { Logarithmic likelihood } \\
\text { value }\end{array}$ & 3978.4 & 3845.2 & 4093.1 & 5798.4 & 6530.5 & 7891.4 & 7993.5 \\
\hline $\begin{array}{l}\text { Adjusted R3 } \\
\text { LR }\end{array}$ & 0.336 & & & & & & \\
\hline
\end{tabular}

Note: The values in brackets are $t$-test values, and $*, * *$, and $* * *$ are significant at the levels of $10 \%, 5 \%$, and $1 \%$, respectively.

Based on the above model 5, this paper measures the development efficiency of the cultural industries in 12 prefecture-level cities in Hubei Province to obtain the efficiency value of the development of the regional cultural industries in Hubei Province from 2003 to 2016. The specific results are shown in table 2.

It can be seen from table 2 that the growth rate of the development efficiency of the cultural industries in Hubei Province from 2003 to 2016 remained at a low level with its average development efficiency value of only 0.4397. From the perspective of various regions, only the average development efficiency of the cultural industries in Wuhan, Ezhou, Jingmen, Huanggang and Suizhou was higher than the average level of the province, but that of the other seven prefecture level cities was lower than the average level. In addition, there was an obvious difference in the development efficiency of the cultural industries in different regions. The average development efficiency of the cultural industries in Shiyan was 0.3022, ranking at the bottom and being only $45.28 \%$ of the average level of Wuhan which ranked top. 
Evaluating the Development Efficiency of Cultural Industry by a Bilateral SFA Model

Table 2. Development efficiency of the cultural industries in Hubei Province from 2003 to 2016

\begin{tabular}{|c|c|c|c|c|c|c|c|c|c|}
\hline Region & 2003 & 2004 & 2005 & 2006 & 2007 & 2008 & 2009 & 2010 & 2011 \\
\hline Wuhan & 0.5381 & 0.7258 & 0.8801 & 0.8222 & 0.831 & 0.7844 & 0.7035 & 0.9517 & 1.0103 \\
\hline Huangshi & 0.423 & 0.2953 & 0.2942 & 0.2845 & 0.359 & 0.4039 & 0.3261 & 0.5111 & 0.5323 \\
\hline Shiyan & 0.2472 & 0.3143 & 0.3254 & 0.3647 & 0.3008 & 0.2595 & 0.2681 & 0.3257 & 0.3288 \\
\hline Yichang & 0.509 & 0.5159 & 0.4287 & 0.3959 & 0.3859 & 0.3856 & 0.3916 & 0.4783 & 0.4831 \\
\hline Xiangyang & 0.3735 & 0.4175 & 0.4186 & 0.3126 & 0.2536 & 0.1991 & 0.2527 & 0.2903 & 0.3675 \\
\hline Ezhou & 0.4182 & 0.4538 & 0.4694 & 0.436 & 0.4261 & 0.4065 & 0.3884 & 0.5114 & 0.5444 \\
\hline Jingmen & 0.389 & 0.7491 & 0.6063 & 0.8645 & 0.9558 & 0.8167 & 0.6983 & 0.8927 & 0.9111 \\
\hline Xiaogan & 0.326 & 0.3483 & 0.3828 & 0.277 & 0.2806 & 0.255 & 0.2924 & 0.3629 & 0.3526 \\
\hline Jingzhou & 0.5151 & 0.6286 & 0.6056 & 0.2926 & 0.357 & 0.319 & 0.2483 & 0.3504 & 0.3026 \\
\hline Huanggang & 0.4257 & 0.4942 & 0.5181 & 0.5366 & 0.5459 & 0.6083 & 0.6177 & 0.719 & 0.7546 \\
\hline Xianning & 0.2701 & 0.4801 & 0.439 & 0.38 & 0.3651 & 0.3711 & 0.2878 & 0.4915 & 0.5872 \\
\hline Suizhou & 0.3852 & 0.5401 & 0.5104 & 0.4701 & 0.5009 & 0.474 & 0.4289 & 0.5633 & 0.5816 \\
\hline Mean value & 0.4017 & 0.4969 & 0.4899 & 0.4531 & 0.4635 & 0.4403 & 0.4087 & 0.5374 & 0.563 \\
\hline Region & 2012 & 2013 & 2014 & 2015 & 2016 & $\begin{array}{c}\text { Mean } \\
\text { value }\end{array}$ & & & \\
\hline Wuhan & 0.4141 & 0.3825 & 0.5248 & 0.3756 & 0.3993 & 0.6674 & & & \\
\hline Huangshi & 0.5501 & 0.4745 & 0.3752 & 0.333 & 0.3046 & 0.3905 & & & \\
\hline Shiyan & 0.3937 & 0.3846 & 0.3225 & 0.188 & 0.2079 & 0.3022 & & & \\
\hline Yichang & 0.4168 & 0.3823 & 0.2307 & 0.2087 & 0.2896 & 0.393 & & & \\
\hline Xiangyang & 0.4437 & 0.406 & 0.3633 & 0.2763 & 0.3004 & 0.3339 & & & \\
\hline Ezhou & 0.4709 & 0.4537 & 0.4026 & 0.4169 & 0.4067 & 0.4432 & & & \\
\hline Jingmen & 0.4365 & 0.3705 & 0.6036 & 0.6427 & 0.6211 & 0.6827 & & & \\
\hline Xiaogan & 0.3287 & 0.3358 & 0.3788 & 0.409 & 0.4232 & 0.3395 & & & \\
\hline Jingzhou & 0.2347 & 0.2565 & 0.3278 & 0.3192 & 0.323 & 0.3629 & & & \\
\hline Huanggang & 0.2907 & 0.2427 & 0.3386 & 0.3534 & 0.3489 & 0.4853 & & & \\
\hline Xianning & 0.3227 & 0.3014 & 0.4122 & 0.4311 & 0.3977 & 0.3955 & & & \\
\hline Suizhou & 0.378 & 0.5016 & 0.5253 & 0.441 & 0.4206 & 0.4801 & & & \\
\hline Mean value & 0.3901 & 0.3743 & 0.4005 & 0.3662 & 0.3703 & 0.3901 & & & \\
\hline
\end{tabular}

From the perspective of the time changing trends, the development efficiency of the cultural industries in Hubei has shown a volatile growth on the whole. It grew with fluctuations in the range between 0 and 0.5 from 2003 to 2009; fluctuated between 0.5 and 0.6 from 2010 to 2011; declined to between 0.3 and 0.4 from 2012 to 2016 . It grew steadily from 2003 to 2009 ; experienced a short period of high growth from 2010 to 2011; and declined from 2012 to 2016.

From the perspective of various regions, Wuhan led the whole province in terms of the development of the cultural industries. Jingmen and Suizhou, which are well known.

Historical and cultural cities, ranked ahead of Wuhan and top three in Hubei. Due to its geographical location, Shiyan's development efficiency was at the bottom in the province. From the perspective of the changing trend of the development efficiency of the cultural industries in various regions, most prefecture-level cities showed the trend of fluctuating growth and were rising on the whole; and the growth fluctuated due to macroeconomic fluctuations in some years. 


\section{Xiaofei Liu, Wenrui Li}

Based on the panel data of prefecture-level cities in Hubei Province from 2003 to 2016, this paper adopts the SFA model and Cobb-Douglas production function to measure the development efficiency of the cultural industries in 112 prefecture-level cities in Hubei Province. The paper has achieved the conclusions as follows: (1) the development efficiency growth of the cultural industries in Hubei Province has been at a low level. Among which, the average development efficiency of the cultural industries in Wuhan was at the highest level and that of Shiyan was at the lowest level. (2) There were significant differences among the regions. Only the average development efficiency of the cultural industries in Wuhan, Ezhou, Jingmen, Huanggang and Sui-zhou was higher than the average level of the province, but that of the other seven prefecture-level cities was lower than the average level of the province. (3) There were obvious fluctuations in terms of time trends. The development efficiency grew steadily from 2003 to 2009; experienced a short period of high growth from 2010 to 2011; and declined from 2012 to 2016.

\section{Conclusions and Suggestions}

5.1 To accelerate the construction of the cultural talent team and improve the mechanism of capital investment in the cultural industries.It is suggested that the Central China may highlight the strategy of strengthening the province by talents, improve the level of human capital in the cultural industries by training and introducing cultural leaders, and continuously improve the level of education of personnel in the cultural industries, thereby enhancing the connotative development capability and driving force of the cultural industries. Therefore, it is necessary to implement full union with universities and colleges to provide a continuous supply of nutrients for human resources of the cultural industries by making full use of the universities and colleges to train high-quality talents, and using various types of onthe-job training and other methods. The Central China should increase investment in the cultural industries and play a cluster effect through a rational allocation of capital and talents, so as to improve the development efficiency of the cultural industries in the Central China.

5.2To accelerate the adjustment of economic structure and promote the supply-side reform of the cultural industries. It is recommended to promote the transformation of the Central China's economic development momentum from one-sided dependence on foreign trade and investment in the past to paying more attention to domestic demand and residents' consumption demand. In order to meet diversified needs, it is necessary to change the traditional ideas for developing the cultural industries in the past and continuously explore new creative ideas in the cultural industries, so as to enhance the attraction of the cultural industries and products to customers. the Central China may improve the total factor productivity of the cultural industries through transforming the cultural supply from being homogeneous to being highquality, and combine it with various superior cultural resources to achieve the goal of reducing the institutional transaction costs of the cultural industries and improving the development efficiency of the cultural industries. the Central China should promote the strategic adjustment of the structure of the cultural industries in the 
context of "Internet+culture", strive to play the role of cultural resource optimization and integration in the allocation of production factor resources, and integrate multiparty resources and strengthen mutual cooperation in multiple links including cultural product, creation, production, marketing, etc.

5.3 To accelerate the integrated development of the cultural industries in the urban and rural areas and encourage the development of private cultural enterprises. Due to the improper allocation of cultural resources between urban and rural areas, it is necessary to establish a mechanism for the development of the cultural industries linking urban and rural areas, so as to rationally allocate resources and improve the efficiency of cultural resource allocation. From the perspective of economic development efficiency, private cultural enterprises are relatively more efficient in development, because they are less restricted by policies and systems and can better play their initiative to carry out cultural industry development according to their own business rules. Therefore, the Central China should vigorously support a number of large scale and special private cultural enterprises to achieve diversified development of regional cultural industries.

5.4 To improve the integration of cultural industries and tourism. The Central China should vigorously develop the tourism industry and rely on its regional cultural advantages to improve the popularity of local culture through the advertising of the tourism industry development. In this process, the Central China should, on the one hand, continuously dig the humanistic connotation of local cultural traditions and attach economic meaning to the cultural connotation to implant it into the whole process of the tourism industry development. On the other hand, it should give full play to the advantages of various cultural resources and combine the characteristics of regional cultural resources to develop new tourism products and characteristic tourism projects with cultural symbols to enhance the taste and creativity of the tourism industry development. Moreover, the Central China should make full use of the driving force of local cultural festivals and other traditional folk activities to accelerate the transformation of festivals and folk activities through protecting traditional festivals and folk activities under the premise of maintaining the original characteristics of traditional folk customs, so as to adapt to the market demand for cultural products.

5.5 To enhance the integration of technological innovation and cultural industry development. To develop the cultural industries, it is necessary to follow the basic laws of economic development. The innovative development of cultural technology can be promoted through establishing the system of cultural technology innovation. Among which, enterprises are the main carriers of cultural technology innovation; market demands are the main focus of cultural technology innovation; while in-depth industry-university-research cooperation is the main means for cultural technology innovation. In order to realize the innovation of the scientific and technological culture industry, it is necessary to continuously improve the level of cultural industry cluster. The Central China should attract the settlement of a large number of diversified cultural enterprises and cultural and creative companies in the region and give full play to the sharing and communication of cultural innovation knowledge 


\section{Xiaofei Liu, Wenrui Li}

brought by the cluster to improve the vitality of cultural technology innovation and promote the innovative and integrated development of the regional cultural industries.

\section{REFERENCES}

[1]Evgallığlu A. E., Gencer C. (2017), Efficiency Measurement of Turkish Natural Gas Distribution Companies by Using Stochastic Frontier Analysis [C]//3 rd International Researchers; Statisticians and Young Statisticians Congress 24-26 May Selcuk University;

[2] Ahmad M. I.(2017), Technical Efficiency and Its Determinants: A Stochastic Frontier Analysis of Sugar Mills in Uttar Pradesh[J].IUP Journal of Applied Economics, 16(4);

[3] Pross C., Strumann C., Geissler A., et al. (2018), Quality and Resource Efficiency in Hospital Service Provision: A Geo Additive Stochastic Frontier Analysis of Stroke Quality of Care in Germany[J]. PloS One, 13(9): e0203017; [4] Herrero-Prieto L. C., Gomez-Veg (2017),Cultural Resources as a Factor in Cultural Tourism Attraction: Technical Efficiency Estimation Of Regional Destinations in Spain[J]. Tourism Economics, 23(2): 260-280;

[5] Figueroa V., Herrero L. C., Báez A., et al. (2018), Analysing How Cultural Factors Influence the Efficiency of Tourist Destinations in Chile[J].International Journal of Tourism Research, 20(1): 11-24;

[6] Li XH, Chen XH,(2013),Research on the Measurement of Development Efficiency of the Cultural Industries in China, 2000 - 2011 [J], China Soft Science, (1):143-148.

[7] Zhao Q., Yang XiuY., Lei Y., et al. (2015), Development Efficiency of the Cultural and Creative Industry in China: Research on Industry Differences and Influencing Factors [J]; Inquiry into Economic Issues,(11):88-97;

[8] Dong Y.J., (2012), Research on the Factors Influencing the Efficiency of Regional Cultural Industries - Analysis Based on the Stochastic Frontier Analysis Model [J]; Journal of Business Economics, (7):29-39;

[9]Ma Y.R., Bai Y., Cheng W.B. (2012), Analysis of the Efficiency and Influencing Factors of China's Cultural Industries Based on SFA [J].Statistics \& Decision,(8):97-101;

[10] Zhao Y., Wei J., ( 2015), Research on the Development Efficiency of Regional Cultural Industries in China - From the Perspective of the Stochastic Frontier Analysis Model [J]. Research on Financial and Economic Issues,(1)30-36; [11]Battese G. E., Corra G. S. (1977), Estimation of a Production Frontier Model: With Application to the Pastoral Zone of Eastern Australia[J]. Australian Journal of Agricultural Economics, 21(3):169-179;

[12]Kumbhakar, Subal C.(2000), Estimation and Decomposition of Productivity Change When Production Is Not Efficient: A Panel Data Approach[J].

Econometric Reviews, 19(4):312-32. 